\title{
OPEN Polycrystalline texture causes magnetic instability in greigite
}

\author{
Barbara Lesniak ${ }^{1}$, Dimitrios Koulialias ${ }^{1}$, Michalis Charilaou ${ }^{2}$, Peter G. Weidler ${ }^{3}$, \\ Jordan M. Rhodes ${ }^{4}$, Janet E. Macdonald ${ }^{4}$ \& Andreas U. Gehring ${ }^{1 \bowtie}$
}

Magnetic stability of iron mineral phases is a key for their use as paleomagnetic information carrier and their applications in nanotechnology, and it critically depends on the size of the particles and their texture. Ferrimagnetic greigite $\left(\mathrm{Fe}_{3} \mathrm{~S}_{4}\right)$ in nature and synthesized in the laboratory forms almost exclusively polycrystalline particles. Textural effects of inter-grown, nano-sized crystallites on the macroscopic magnetization remain unresolved because their experimental detection is challenging. Here, we use ferromagnetic resonance (FMR) spectroscopy and static magnetization measurements in concert with micromagnetic simulations to detect and explain textural effects on the magnetic stability in synthetic, polycrystalline greigite flakes. We demonstrate that these effects stem from inter-grown crystallites with mean coherence length (MCL) of about $20 \mathrm{~nm}$ in single-domain magnetic state, which generate modifiable coherent magnetization volume (CMV) configurations in the flakes. At room temperature, the instability of the CVM configuration is exhibited by the angular dependence of the FMR spectra in fields of less than $100 \mathrm{mT}$ and its reset by stronger fields. This finding highlights the magnetic manipulation of polycrystalline greigite, which is a novel trait to detect this mineral phase in Earth systems and to assess its fidelity as paleomagnetic information carrier. Additionally, our magneto-spectroscopic approach to analyse instable $\mathrm{CMV}$ opens the door for a new more rigorous magnetic assessment and interpretation of polycrystalline nano-materials.

Greigite $\left(\mathrm{Fe}_{3} \mathrm{~S}_{4}\right)$ is a ferrimagnetic thio-spinel isomorphic to magnetite, the archetypical mineral in natural magnetism. Since the discovery of greigite in Miocene lake sediments by Skinner et al. ${ }^{1}$, it has been widely used as powerful sulfidic proxy for geochemical reconstructions and as a remanence carrier to record paleomagnetic information ${ }^{2-6} . \mathrm{Fe}_{3} \mathrm{~S}_{4}$ nano-particles have also attracted considerable attention owing to the morphology, and the magnetic and electronic properties ${ }^{7-11}$ for technical applications in areas such as spintronics ${ }^{12}$, catalysis ${ }^{13}$, and biomedicine ${ }^{14}$. Greigite in nature generally forms nano-sized crystallites with variable morphologies, which can aggregate in clumps and nodules ${ }^{5,15-17}$, and similar to magnetite, nano-sized greigite can also form intracellular chain assemblies in magnetotactic bacteria ${ }^{18-20}$. Laboratory studies showed that the morphology of greigite nanoparticles and the formation of assemblies can be tailored by the synthesis process, e.g., by the selection of the organosulfur precursor ${ }^{21,22}$ and that the architecture of the assemblies influences the magnetic properties ${ }^{23}$. In contrast to the oxide counterpart magnetite, the fundamental magnetic properties and especially its size dependence in the nano-meter scale have not been conclusively resolved for greigite and this may limit numerical approaches to infer the physical characteristics $9,12,24-26$. For pure synthetic, $\mu \mathrm{m}$-sized, cubo-octahedreal crystals with a saturation magnetization $M_{\mathrm{s}} \approx 60 \mathrm{Am}^{2} \mathrm{~kg}^{-1}$ was reported whereas the Curie temperature $\left(T_{\mathrm{c}}\right)$ remained ill-defined due to thermal phase instability ${ }^{5,26,27}$. Ferromagnetic resonance (FMR) spectroscopy was applied to constrain the magnetocrystalline anisotropy constant $\mathrm{s}^{24}$. These reported magnetic parameters were obtained from polycrystalline particles, and importantly, the coalescence effects of crystallites were not considered ${ }^{5,27,28}$. Such effect may generate magnetization textures with competitive single crystallite anisotropy properties and their interactions can critically affect the size and stability of coherent magnetic volumes (CMV ${ }^{29,30}$. The impact of nano-texturization on the magnetic properties is vital to assess the fidelity of greigite for paleomagnetic studies of the Earth's history and the use of the polycrystalline greigite for recording or technical applications. Here, we present a magneto-spectroscopic approach that detects CMV changes in polycrystalline greigite flakes, providing a new perspective to decipher the magnetic stability of nano-textured Earth materials.

${ }^{1}$ Department of Earth Sciences, Institute of Geophysics, ETH Zurich, 8092 Zurich, Switzerland. ${ }^{2}$ Department of Physics, University of Louisiana at Lafayette, Lafayette, LA 70504, USA. ${ }^{3}$ Institute of Functional Interfaces, Karlsruhe Institute of Technology, 76021 Karlsruhe, Germany. ${ }^{4}$ Department of Chemistry, Vanderbilt University and Vanderbilt Institute for Nanoscale Science and Engineering, Nashville, TN 37235, USA.『email: agehring@ ethz.ch 
a

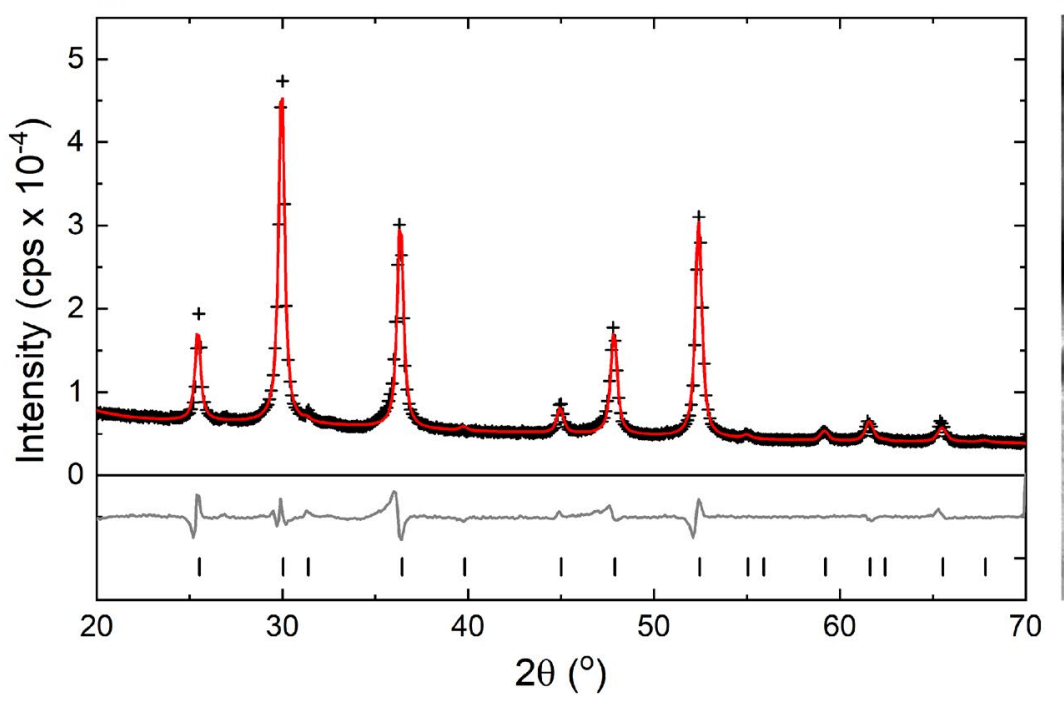

b

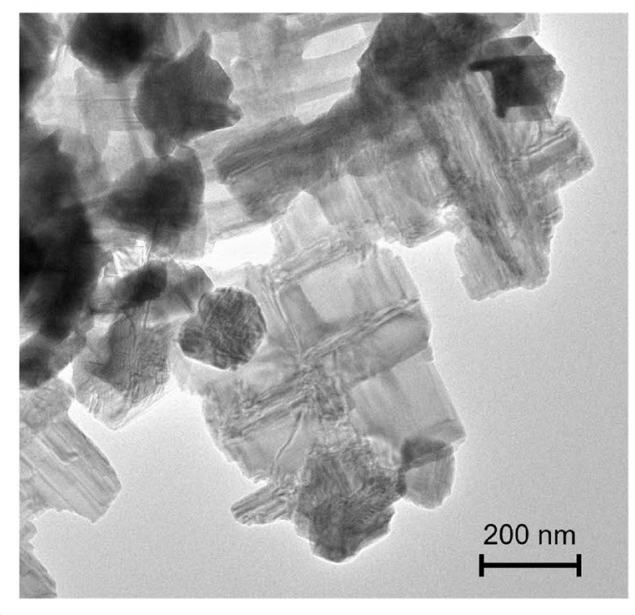

Figure 1. Characterisation of the synthesized greigite sample. (a) powder X-ray diffractogram with measured data point (+), fitted data (red line) and the difference between measurement and model (grey line). (b) TEM picture showing the flake morphology of the greigite.

\section{Results and discussion}

Structural properties. X-ray diffractometry (XRD) exhibits peaks that can be exclusively attributed to greigite, i.e., the synthesized sample is a pure mineral single-phase (Fig. 1a). Moreover, the background of the XRD pattern clearly indicates that the amount of X-ray amorphous constituents is negligible. The Rietveld refinement converged with a goodness of fit of 4.53 and the $\mathrm{R}_{\mathrm{Bragg}}$ of 1.007 . The obtained lattice parameter $a=0.98580 \pm 0.00016 \mathrm{~nm}$ is in excellent agreement with the literature values for greigite ${ }^{1}$. In addition, the mean coherence length (MCL) is $19 \pm 2 \mathrm{~nm}$ and the stress/strain parameter $\varepsilon_{\mathrm{o}}$ is low with 0.00002 for our sample. Transmission electron microscopy (TEM) of the synthesized greigite powder reveals agglomerated nanoparticles in a size range generally between 100 and $200 \mathrm{~nm}$ with rectangular flake morphologies (Fig. 1b). This size range and the MCL inferred from XRD indicate polycrystalline flakes with textures formed by intergrown nanocrystallites. Such polycrystalline composite was reported for nano-sized ${ }^{31}$ and larger greigite particles ${ }^{5}$. Furthermore, the absence of significant strain/stress suggests that the polycrystallinity of the flakes most likely stems from crystal growth and is not a mechanical effect. With this in mind polycrystalline flakes can be considered as the result of the intergrowth of crystallites along variable principle crystallographic axes.

Magnetic properties. At room temperature, the hysteresis loop in a $3 \mathrm{~T}$ field of the polycrystalline flakes shows $M_{3 \mathrm{~T}}=34.2 \mathrm{Am}^{2} \mathrm{~kg}^{1}$, a remanence $M_{\mathrm{r}}=18.4 \mathrm{Am}^{2} \mathrm{~kg}^{-1}, M_{\mathrm{r}} / M_{1 \mathrm{~T}}=0.54$, and a coercivity $\mu_{0} H_{c}=38.2 \mathrm{mT}$ (Fig. 2a). In a $1 \mathrm{~T}$ field the hysteresis parameters are nearly the same with $M_{1 \mathrm{~T}}=32.7 \mathrm{Am}^{2} \mathrm{~kg}^{-1}, M_{\mathrm{r}}=17.7 \mathrm{Am}^{2} \mathrm{~kg}^{-1}$, a coercivity $\mu_{0} H_{c}=41.2 \mathrm{mT}$ and a coercivity of remanence $\mu_{0} H_{c r}=54.8 \mathrm{mT}$. The squareness ratio $M_{\mathrm{r}} / M_{1 \mathrm{~T}}=0.54$ and $H_{c r} / H_{c}=1.33$ are indicative of uniaxial single domain (SD) particles ${ }^{32}$. The corresponding first order reversal curve (FORC) contour plot has an oval shape with a nearly symmetric $\mu_{0} H_{c}$ distribution in the range of 10 and $100 \mathrm{mT}$ with a maximum at about $50 \mathrm{mT}$ that is in accordance with SD greigite (Fig. 2b). Moreover, the contour plot is shifted to the negative internal bias field $\left(H_{\mathrm{u}}\right)$, which has been interpreted as a magnetostatic interaction effect $^{32}$. It is worth noting that $\mathrm{Hu}$ et al..$^{5}$ previously mentioned that the SD magnetic state of greigite particles in the $100 \mathrm{~nm}$ order of magnitude stems from their polycrystalline texture with nano-sized MCL. The thresholds of the magnetic domain states for greigite are poorly constrained. Micromagnetic modelling, however, suggests that greigite crystallites with MCL of about $20 \mathrm{~nm}$ are likely in SD magnetic state and that their pseudo-single domain/multidomain (PSD/MD) threshold is about $200 \mathrm{~nm}^{11}$. Given this, the magnetization of polycrystalline flakes with sizes of about $200 \mathrm{~nm}$ (Fig. 1b) can be considered as the sum of the magnetizations of intergrown SD crystallites.

The saturation magnetization $\left(M_{\mathrm{s}}\right)$ of greigite reported in the literature varies and the highest value $M_{\mathrm{s}} \approx 60$ $\mathrm{Am}^{2} \mathrm{~kg}^{-1}$ was found for $\mu \mathrm{m}$-sized, cubo-octahedral crystals ${ }^{8,26}$. Synthetic and natural nano-sized greigite reveal much lower apparent $M_{\mathrm{s}}$ of generally less than $40 \mathrm{Am}^{2} \mathrm{~kg}^{-128}$. Considering the XRD pattern and its background, it is very unlikely that the relatively low value $M_{1 \mathrm{~T}}=32.7 \mathrm{Am}^{2} \mathrm{~kg}^{-1}$ of our sample is due to an additional sulfide phase or X-ray amorphous constituents. Therefore, the departure from the expected $M_{\mathrm{s}}$ is most probably caused by inhomogeneities, i.e., at the surfaces and interfaces of the crystalites ${ }^{33}$. It is worth noting that a similar observation was reported for polycrystalline greigite nanoparticles with MCL of $20 \mathrm{~nm}^{28}$.

Low-temperature measurements exhibit continuous development of the hysteresis parameters with $M_{3 \mathrm{~T}}=37.5$ $\mathrm{Am}^{2} \mathrm{~kg}^{-1}$ and $\mu_{0} H_{c}=75 \mathrm{mT}$ at $10 \mathrm{~K}$, but the corresponding change in $M_{\mathrm{r}} / M_{3 \mathrm{~T}}$ is marginal (Fig. 2a). A similar $H_{c}$ behaviour was reported by Coey et al. ${ }^{34}$ for a nano-sized polycrystalline greigite powder. Such behaviour and 
a

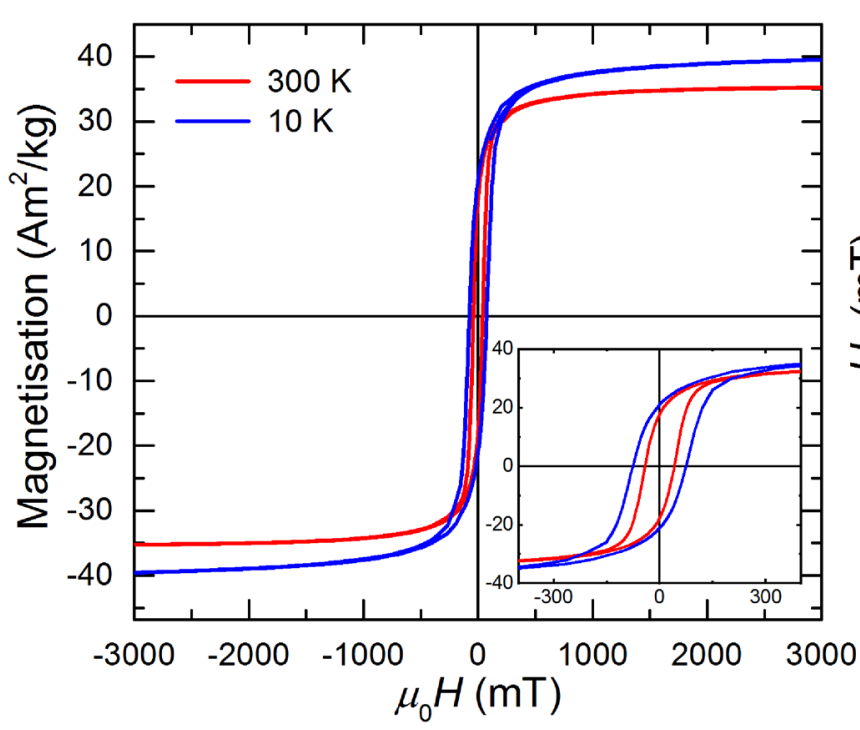

b

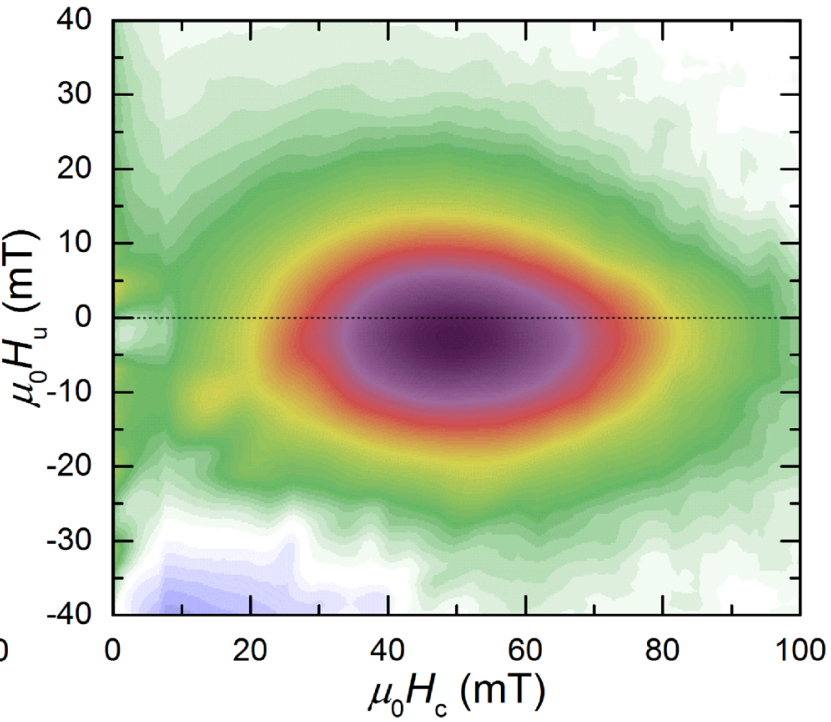

Figure 2. Magnetic hysteresis properties. (a) $M(H)$ plots at $300 \mathrm{~K}$ and $10 \mathrm{~K}$ and enlarged hysteresis loops (inset). (b) FORC diagram obtained by processing of magnetic data recorded in fields up to $1 \mathrm{~T}, \mathrm{SF}=8$; with nearly symmetric $H_{\mathrm{c}}$ distribution at $H_{\mathrm{u}}=0$ with highest values in dark purple.
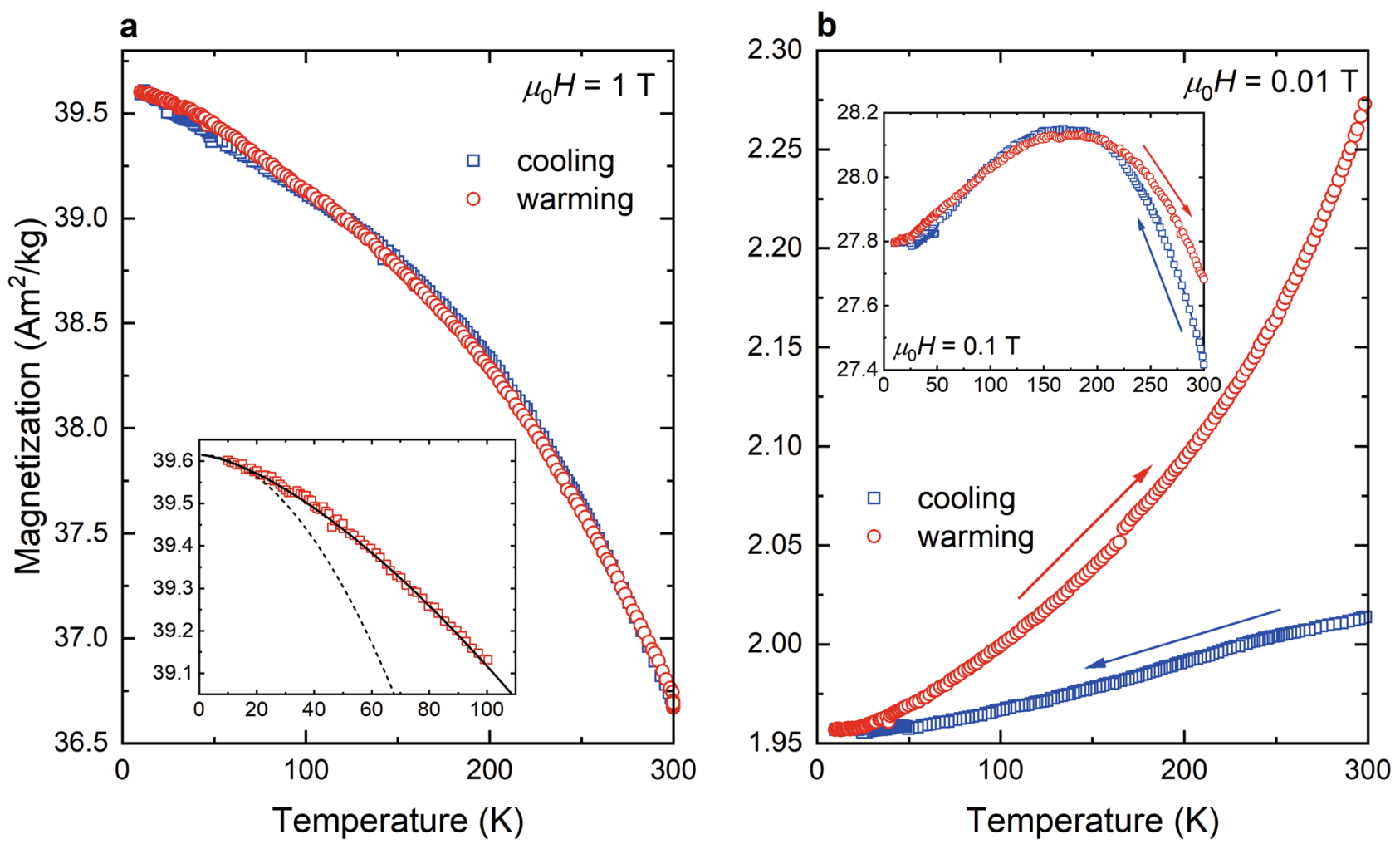

Figure 3. Low-temperature magnetization cycling. (a) Curves recorded in a $1 \mathrm{~T}$ field with inset shows the fit using the Bloch law with non-constraining $T_{\mathrm{c}}$ (solid line), dashed line with $T_{\mathrm{c}}=600 \mathrm{~K}$ (see text). (b) curves recorded in a $10 \mathrm{mT}$ field and a $100 \mathrm{mT}$ field (inset).

the nearly constant $M_{\mathrm{r}} / M_{3 \mathrm{~T}}$ ratio confirm that greigite has no low-temperature anomaly as it is known for the isomorphic magnetite with its Verwey transition ${ }^{27,34}$.

Magnetization cycling experiments at low temperature reveal field-dependent behaviour (Fig. 3). Congruent cooling and warming curves were found for $\mu_{0} H \geq 0.5 \mathrm{~T}$ (Fig. 3a). In a field of $1 \mathrm{~T}$ that apparently saturates the bulk of the greigite crystallites (Fig. 2a), the curves can be fitted with the Bloch law $M(T)=M_{0}\left(1-\left(T / T_{c}\right)^{3 / 2}\right)$, where $M_{0}$ is the magnetization at $T=0$ and $T_{\mathrm{c}}$ is the Curie temperature (Fig. 3a). Considering the estimated $T_{\mathrm{c}} \approx$ 
a

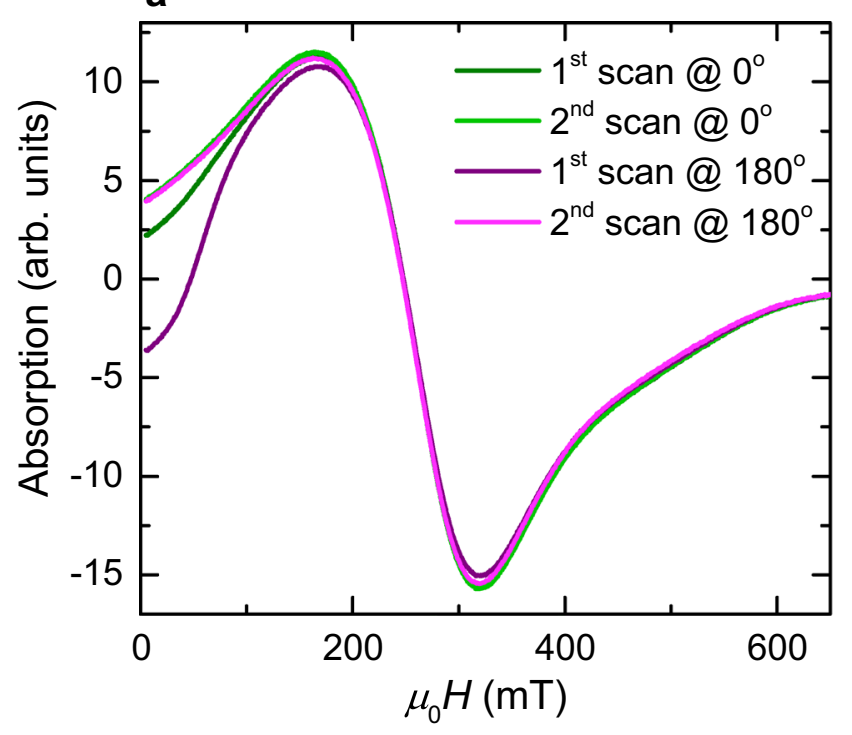

b

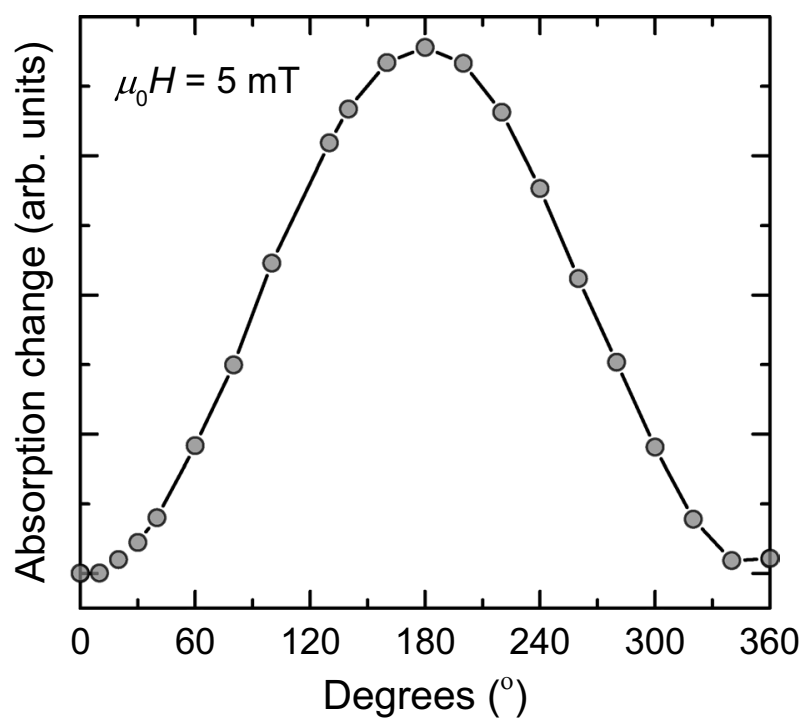

Figure 4. Rotational behaviour of FMR spectra at $300 \mathrm{~K}$. (a) Initial and second spectra in $0^{\circ}$ positions and first and second spectra in $180^{\circ}$ positions with varying absorption behaviours in the low-field range at $\mu_{0} H<200$ $\mathrm{mT}$ and with second scans superimposed. (b) Angular effect of the absorption in $\mu_{0} H=5 \mathrm{mT}$ depicted as the departure from the reference absorption [2nd scans shown in (a)] in absolute values.

$600 \mathrm{~K}$ for greigite ${ }^{26} M$ follows the $T^{3 / 2}$ law only in a temperature range between 5 and $20 \mathrm{~K}$ (Fig. 3a inset). Fitting over a wider temperature range results in unrealistic $T_{\mathrm{c}}>1000 \mathrm{~K}$. Chang et al. ${ }^{26}$ reported a similar behaviour for synthetic, $\mu \mathrm{m}$-sized greigite particles with $M_{\mathrm{s}} \approx 60 \mathrm{Am}^{2} \mathrm{~kg}^{1}$. The cycling experiment with $\mu_{0} H=0.5 \mathrm{~T}$, however, also reveals reversible magnetic behaviour of the cooling and warming curves. Irreversibility occurs in magnetic fields between 10 and $100 \mathrm{mT}$, which correspond to the coercivity range found in the FORC diagram (Figs. 2b, $3 \mathrm{~b})$. This behaviour is relatively weak in $100 \mathrm{mT}$, where the cycling increases the magnetization at $300 \mathrm{~K}\left(\Delta M_{300 \mathrm{~K}}\right)$ by about $1 \%$ (Fig. 3 b, inset). Both curves exhibit a concave shape with a maximum at $T \approx 160 \mathrm{~K}$. Such behaviour is known for pyrrhotite $\left(\mathrm{Fe}_{7} \mathrm{~S}_{8}\right)$ and was explained by the interplay between the Zeeman energy $\left(E_{z}\right)$ and the magnetocrystalline anisotropy energy ${ }^{35}$. For the greigite flakes the departure from the Bloch law, however, can be attributed to the absence of saturation and to the finite size of the crystallites and their interfaces, which affect the local chemical potential ${ }^{36,37}$. To test this interpretation, the cycling experiments were performed in weaker fields, under the assumption that crystallite and interface effects become more pronounced by lowering $E_{z}$. In a field $\mu_{0} H=10 \mathrm{mT}$, the cooling curve exhibits a nearly linear decrease of about $3 \%$ down to $10 \mathrm{~K}$ and a subsequent monotonic increase upon warming that follows a power law (Fig. 3b). After the cycling, the gain in magnetization at $300 \mathrm{~K}\left(\Delta M_{300 \mathrm{~K}}\right)$ is $12.9 \%$. In general, SD particles exhibit similar cooling and warming curves whereas MD particles show demagnetization during the cycling due to domain wall dynamics ${ }^{35,38}$. Amplitudedependent ac susceptibility up to $\mu_{0} H_{\mathrm{ac}} \leq 1.5 \mathrm{mT}$ at $300 \mathrm{~K}$ provides no evidence of domain-wall motions in our polycrystalline greigite flakes, i.e., the domain walls associated with the interfaces are relatively stiff. Given this, the peculiar behaviour of the flakes is probably an extrinsic, textural effect. Studies on nanoparticle ensembles showed that competition between crystallites can critically affect the CMV of the constituents ${ }^{29}$ and consequently the magnetization texture of a sample. Considering the MCL of $19 \pm 2 \mathrm{~nm}$ for our flakes, the $\Delta M_{300 \mathrm{~K}}$ shift to zero with increasing field points to polycrystalline textures with changing CMV. In a near saturation field where $\Delta M_{300 \mathrm{~K}}=0 \%$ the different CMV configurations in the flakes are wiped out. The irreversible magnetization during thermal cycling in $\mu_{0} H \leq 100 \mathrm{mT}$ implies that a weak field modifies the CMV and this in turn the magnetization texture of the flakes.

FMR spectroscopy. FMR spectroscopy can be used to experimentally constrain the interplay between anisotropy and magnetostatic interactions. The spectrum of the polycrystalline flakes at $300 \mathrm{~K}$ is asymmetric with a symmetry ratio $A_{\text {ratio }}=0.84$, i.e., negative skewness ${ }^{39}$, and with spectral parameters $\mu_{0} H_{\text {res }}=247.6 \mathrm{mT}, g=2.85$ and $\mu_{0} \Delta H=155 \mathrm{mT}$ and it exhibits near zero-field absorption (Fig. 4a). The spectra obtained from dispersed powder samples of synthetic, $\mu \mathrm{m}$-sized PSD/MD greigite particles reported by Chang et al. ${ }^{40}$ show quite similar spectral parameters but positive skewness $\left(g=2.95-3.13, \mu_{0} \Delta H \approx 200 \mathrm{mT}\right.$, and $\left.A_{\text {ratio }}>1\right)$. This implies that grain-size and shape of polycrystalline particles have only minor effect on the spectral parameters at $300 \mathrm{~K}$. The different skewness of the spectra most likely stems from the sign of the magnetocrystalline anisotropy constants and/or the orientation of the easy axes ${ }^{24}$. Pronounced near-zero field absorption has been generally reported for relatively large MD particles due to domain wall effects ${ }^{41,42}$. Such effects are unexpected for the greigite flakes, which show a magnetic response in the hysteresis measurements typical of SD state (Fig. 2a,b). Therefore, the near-zero field absorption most likely stems from interfaces between nano-crystallites. The FMR spectra at $300 \mathrm{~K}$, however, reveal angular dependence of the absorption intensity in the range of $\mu_{0} H<200 \mathrm{mT}$ and no angular dependence occurs at higher fields as it is generally expected for powder samples (Fig. 4a). In fields less than $200 \mathrm{mT}$, where 
a

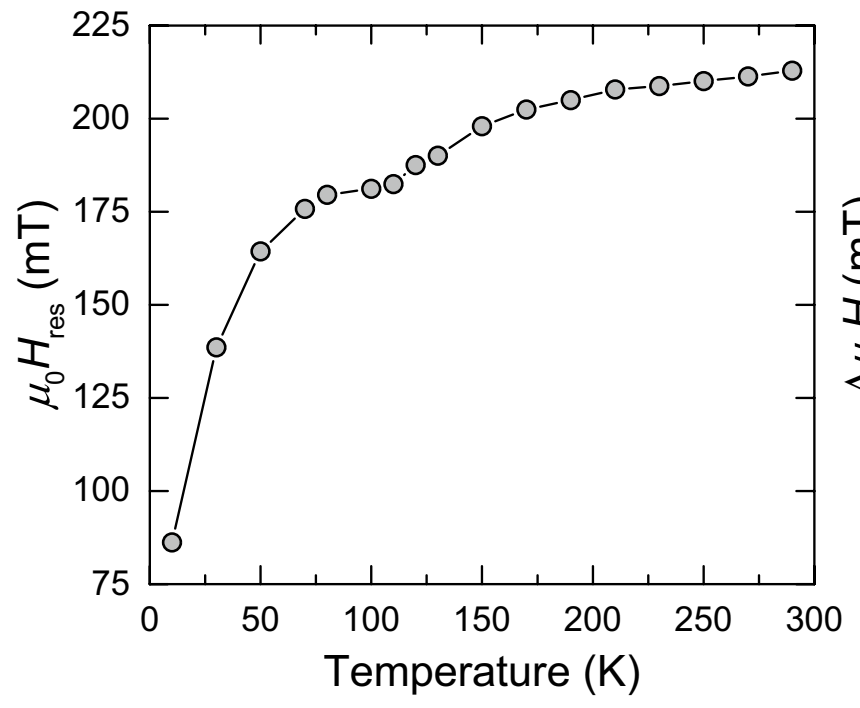

b

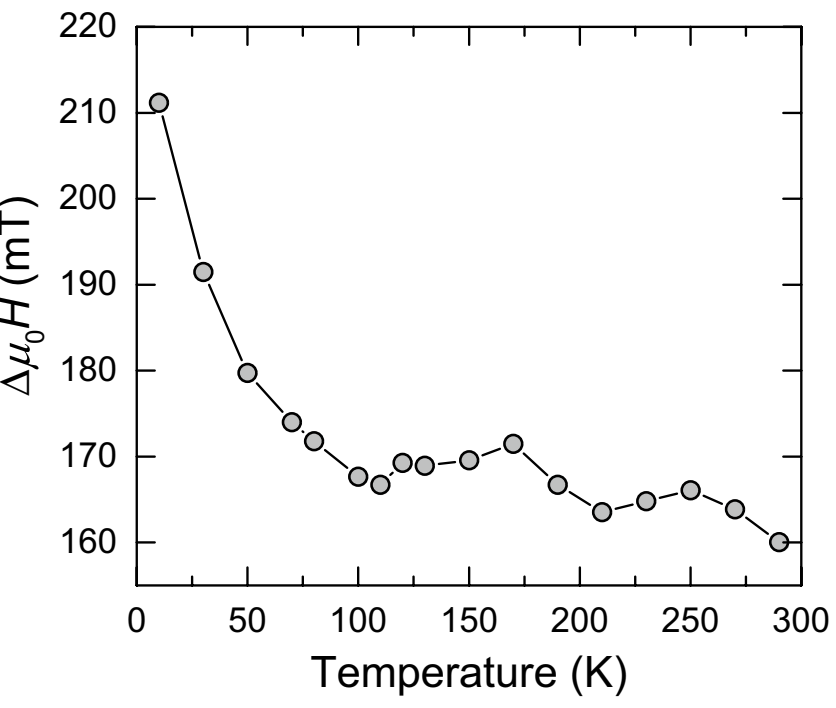

Figure 5. Development of the spectral parameters at low temperatures. (a) $\mu_{0} H_{\text {res }}$ and (b) $\Delta \mu_{0} H$ showing pronounced changes at $T \approx 50 \mathrm{~K}$.

greigite flakes are non-saturated (Fig. 2a), the absorption behaviour changes with the external field orientation and the directional differences tend to zero with increasing fields (Fig. 4a). Moreover, the microwave absorption reveals $2 \pi$ rotational dependence in a low-field range where the configurational entropy is relatively high (Fig. 4b). This points to induced magnetization of the CMV configurations, which are affected by the geometry of the intergrown nano-crystallites, i.e., the angle between their easy axes and the external field. Considering the above, the angular dependence provides clear evidence that in the low-field range, the randomization of the flakes in the sample is broken with respect to the CMV of the polycrystalline matrix. This breaking is annihilated by remagnetization in a sweeping field up to $0.65 \mathrm{~T}$ and the initial FMR response is re-established, i.e., the CMV of the flake exhibits a memory effect.

Figure 5 shows the change of the spectral parameters upon cooling. The resonance field-strength continuously declines down to about $50 \mathrm{~K}$ followed by a prominent decrease and the corresponding increase of the $g$-value to 7.78 at $10 \mathrm{~K}$. A pronounced decrease in $H_{\text {res }}$ is well known for magnetite at the Verwey transition ${ }^{43}$, which is associated with changes of the crystallographic structure from cubic to monoclinic and of the magnetocrystalline anisotropy as documented by a shift of the easy axes from (111) to (100). For such transition in greigite, there is no indication in our magnetization curves (Fig. 3) and also no report in the literature ${ }^{27,34}$. Associated with the pronounced change of $H_{r e s}, \Delta H$ markedly increases without significant change in $A_{\text {ratio }}$. The linewidth broadening points to pronounced increase in interaction energy of the crystallites within the flakes and the simultaneous shift in the $g$-value can be explained by changes in CMV and their anisotropy properties.

The broad spectra recorded at $10 \mathrm{~K}$ show angular dependence that is manifested over the whole sweeping field range (Fig. 6). For the high-field absorption at $\mu_{0} H>150 \mathrm{mT}$ mainly the signal intensity varies. For the low-field range $\mu_{0} H<150 \mathrm{mT}$ the absorptions conspicuously change without a clear angular pattern (Fig. 6). Repeated scans under constant angles generate low-field absorptions that exhibit decreasing intensity with increasing angle between the external field and the sample (Fig. 6). For the high-field absorptions the sweeping field up to $0.65 \mathrm{~T}$ has nearly no effect. Compared to $300 \mathrm{~K}$, the spectral properties at $10 \mathrm{~K}$ imply that at low-temperature stable, inerasable CMV configurations are induced. Considering the low-temperature behaviour of the spectral parameters (Fig. 5), it can be supposed that this effect is more pronounced at $T<50 \mathrm{~K}$. Furthermore, changes in the CMV configurations could explain the peculiar temperature dependence of the magnetization in the cycling experiments (Fig. 3b).

Micromagnetic simulations. In order to obtain additional insight to the magnetic configuration of the greigite flakes, we simulated hysteresis curves for different flake directions and performed micromagnetic simulations of a polycrystalline flake generated by means of Voronoi tessellation (see Figs. 7, 8a). The simulation system in Fig. 8a contains blocks which represent intergrown crystallites with different crystallographic orientations and approximate sizes as obtained by XRD and the colours indicate different directions of the anisotropy vector of each block. The average of the convoluted hysteresis curve of a single flake has a coercivity of $20 \mathrm{mT}$ and remanence of $M_{\mathrm{r}}=0.5 M_{\mathrm{s}}$ (Fig. 7), which are in good agreement with the experimental data obtained from the bulk sample (Fig. 2a). The higher coercivity in the measured $M(H)$ loop is probably due to factors such as anisotropic exchange at the crystallite interfaces and other surface effects that cannot be resolved at this point and are beyond the scope of our micromagnetic model. To account for those contributions, however, atomistic calculations would be required, but the surface/interface parameters for $\mathrm{Fe}_{3} \mathrm{~S}_{4}$ are currently unknown.

Importantly, we find that upon the application of an external field, the CMV start to change by overcoming the crystallite to crystallite interfaces (Fig. 8b-d). At any given time, each crystallite and the CMV, which itself 


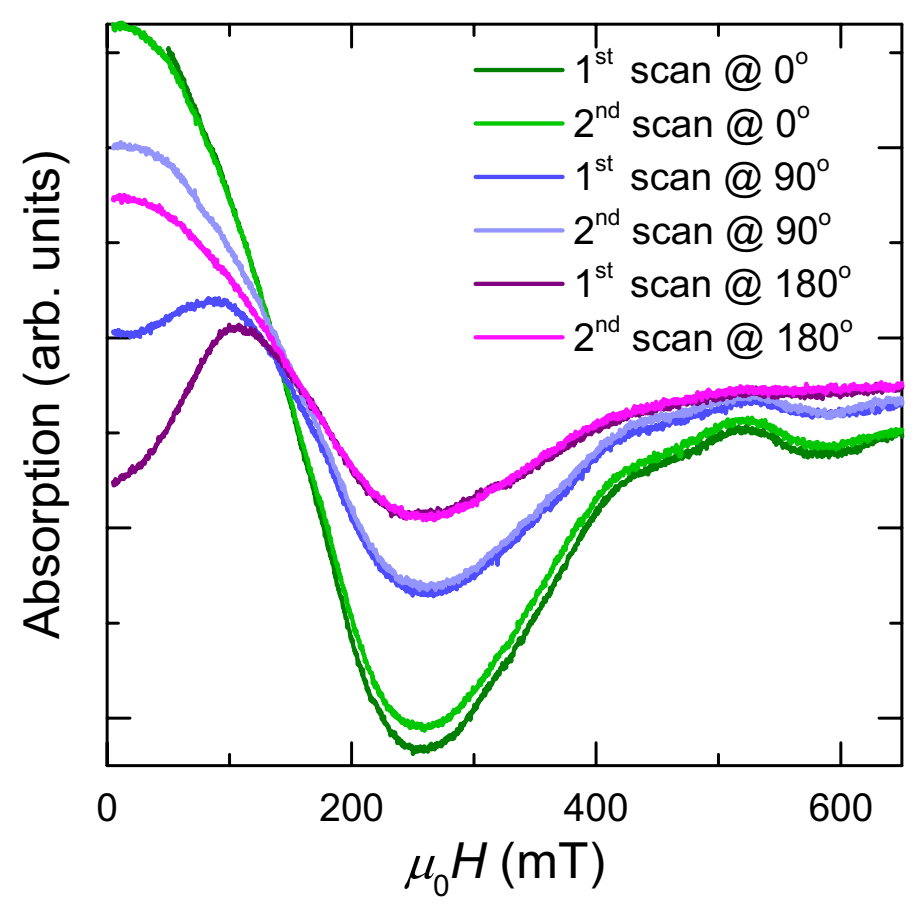

Figure 6. Angular dependence of the FMR spectra at $10 \mathrm{~K}$. First and second scans of the initial spectrum and after rotations by $90^{\circ}$ and $180^{\circ}$, respectively.

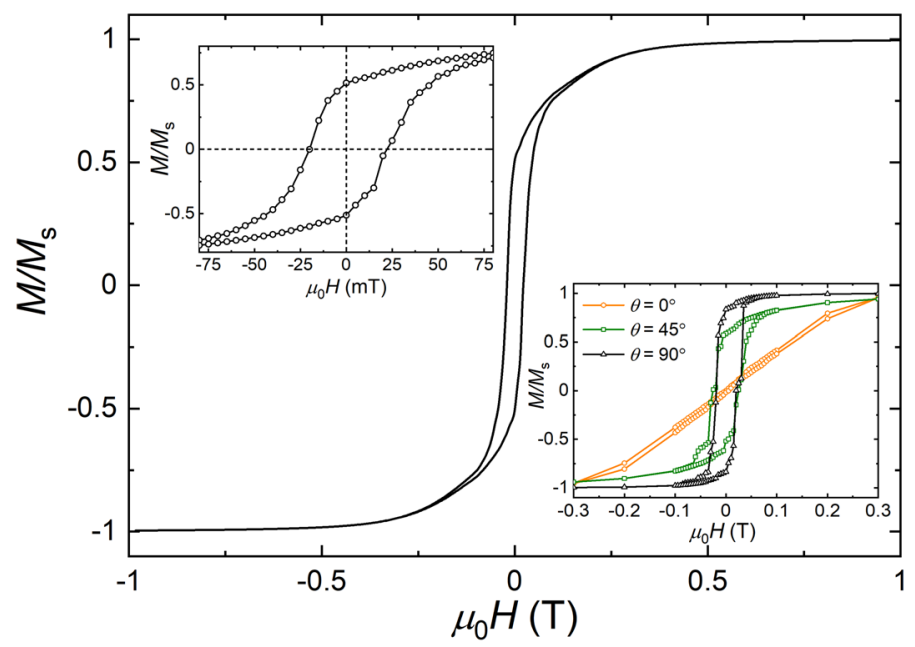

Figure 7. Simulated hysteresis curves. Averaged $M(H)$ curve for different flake directions with the angle $\theta$ from the normal of the flake ranging from $0^{\circ}$ to $90^{\circ}$ with steps of $5^{\circ}$, close-up (left insert) and comparison of selected hysteresis curves yielded with $\theta$ of $0^{\circ}, 45^{\circ}$, and $90^{\circ}$ (right inset).

contains several crystallites, are in a single-domain state, as it is shown by the experimental and simulated coercivity values. The visualisation of the magnetic state obtained from the simulation and its accordance with the experiments $\left(M(H), H_{c}\right)$ support our interpretation of a dominant texture effect on the magnetization process in the flakes. Even though there are some uncertainties of the material parameters $\left(M_{\mathrm{s}}, K, A\right)^{24,26,27}$ the texture effects would remain prominent under the variation of any of those parameters.

Concluding comments. Our finding that field-induced changes of the CMV configuration in polycrystalline greigite can generate a reversible instability is of importance in two respects. First, magnetic stability is crucial for the use of greigite in paleomagnetism. Greigite flakes with nano-sized textures exhibit a memory effect in the $10 \mathrm{mT}$ field range as revealed by FMR spectroscopy. This range, however, is magnitudes higher compared to the Earth's magnetic field of about $0.05 \mathrm{mT}$, and, therefore, such a weak field induces no modifiable CMV configurations. Hence, the fidelity of greigite as natural remanence carrier in paleomagnetism is not 

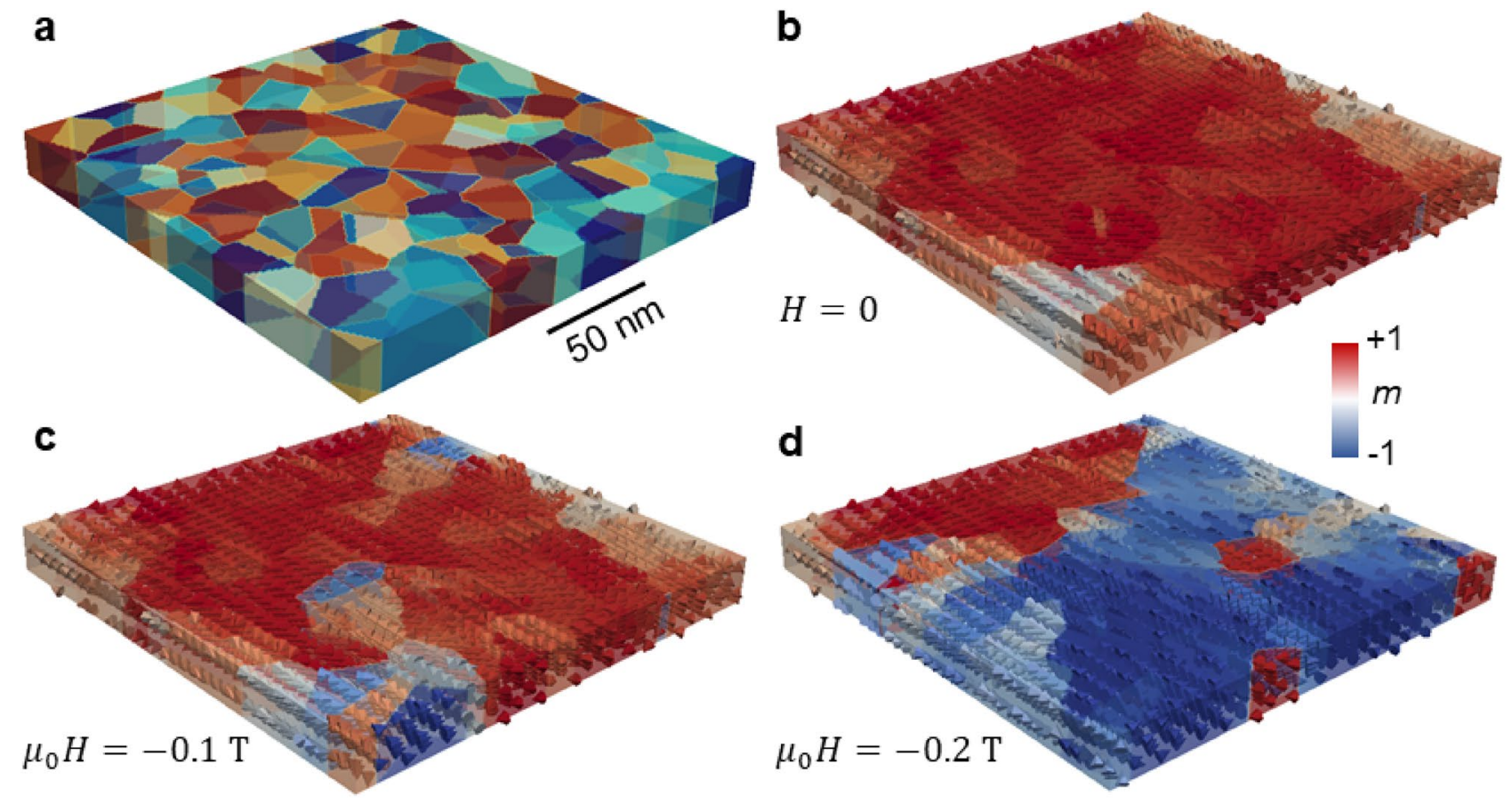

Figure 8. Micromagnetic simulations of a greigite flake. (a) exhibits the simulated system that mimics a greigite flake with dimensions of $200 \mathrm{~nm} \times 200 \mathrm{~nm} \times 20 \mathrm{~nm}$ consisting of intergrown crystallites with a size of about $20 \mathrm{~nm}$ and the colours mirror the different anisotropy vectors. (b) Starting from remanence after saturation, (c,d) the application of an external field generates a CMV configuration.

called into doubt by textural effects as long as they are physio-chemically stable. Moreover, the memory effect is temperature-dependent and generates an induced magnetization behaviour unique among magnetic minerals. This can be utilized as a novel trait in order to detect nano-sized, textured greigite as magnetic proxy to reconstruct sulfidic geological systems.

Second, our finding further demonstrates that polycrystalline greigite flakes can be considered as magnetic nano-composites, where SD, inter-grown nano-crystallites form variable CMV configurations. The CMV are manipulable in the range of the coercivity fields and the induced change in the magnetic texture of the flakes can be reset by higher fields. Such memory effect stemmed from the textural architecture provides an invaluable, experimental basis for continuative, fundamental considerations of the influence of intergrown, nanoscopic crystallites on the magnetization of microscopic particles.

\section{Methods}

Sample preparation. The greigite flakes were synthetized through the phase-controlled colloidal synthesis described by Rhodes et al. ${ }^{21}$. The sample was produced by heating of the mixture of organosulphur precursor dibenzyl disulfide and $\mathrm{FeCl}_{2}$ in the presence of oleylamine. In a $25 \mathrm{~mL}$ 3-neck round bottom flask, $\mathrm{FeCl}_{2}$ $(0.50 \mathrm{mmol})$ and $10 \mathrm{~mL}$ of oleylamine were placed under vacuum for $1 \mathrm{~h}$ at $60^{\circ} \mathrm{C}$. The temperature was increased to $170^{\circ} \mathrm{C}$ and maintained for $1 \mathrm{~h}$ under an inert $\mathrm{N}_{2}$ atmosphere. In a separate vial, dibenzyl disulfide (1.5 mmol) was dissolved in $5.0 \mathrm{~mL}$ of oleylamine and placed under vacuum at room temperature for 5 min followed by backfilling with $\mathrm{N}_{2}$ for $15 \mathrm{~min}$. The dibenzyl disulfide solution was then injected into the reaction flask and heated to $220{ }^{\circ} \mathrm{C}$ for $2 \mathrm{~h}$ under $\mathrm{N}_{2}$. The reaction solution was then cooled in air to room temperature, and $40 \mathrm{~mL}$ if chloroform was added followed by centrifugation for $5 \mathrm{~min}$ at $8000 \mathrm{rpm}$. Particles were further purified by two cycles of suspension with chloroform followed by ethanol (total $20 \mathrm{~mL}$ ), the centrifugation for $5 \mathrm{~min}$ at $4400 \mathrm{rpm}$. The particles were stored in chloroform.

Structural characterization. X-ray diffraction analysis was performed on a Rigaku SmartLab X-ray diffractometer equipped with a $\mathrm{CuK}_{\alpha}$ radiation source and D/teX Ultra 250 detector, operating at $40 \mathrm{kV}$ and $44 \mathrm{~mA}$. XRD samples were prepared by drop casting a concentrated solution of NPs onto a glass holder. The diffractogram was evaluated with the Bruker AXS TOPAS6.0 program ${ }^{44}$ applying the modified Thompson-Cox-Hastings pseudo-Voigt function ${ }^{45}$. The background was modeled by a polynomial of 6 th degree. For the refinement, the structure model by Skinner et al. ${ }^{1}$ was taken. Line shape parameters, the lattice parameter, and global thermal factors for each atomic site were refined. Fractional coordinates and occupation numbers kept untouched. From these fits, the lattice parameters, the MCL size and the stress/strain parameter $\varepsilon_{\mathrm{o}}=\Delta d / d$, where $\Delta d$ is the mean deviation of the lattice spacing $d$ in per mill, were obtained. The size and the shape of synthesized greigite particles were determined by a transmission electron microscope (FEI Tecnai Osiris S/TEM operating at $200 \mathrm{kV}$ ). 
Magnetic and FMR spectroscopic analyses. The magnetic characterization was performed by static and dynamic magnetization experiments at $300 \mathrm{~K}$ and at low temperature. A vibrating sample magnetometer (Princeton Measurement Corporation 3900 MicroMag) and Quantum Design Physical Property Measurement System (PPMS) were used to measure hysteresis properties in a $1 \mathrm{~T}$ field at $300 \mathrm{~K}$ and in a $3 \mathrm{~T}$ field down to $10 \mathrm{~K}$, respectively. The coercivity distribution and internal bias fields in a range up to $1 \mathrm{~T}$ at $300 \mathrm{~K}$ were analysed by FORC diagrams ${ }^{46}$ obtained from 160 curves, increment fields of $1.87 \mathrm{mT}$, an average time of $100 \mathrm{~ms}$ and by using the FORCinel code ${ }^{47}$ with a smoothing factor $\mathrm{SF}=8$ to compute the first-order reversal curve distributions. Moreover, in the temperature range between 300 and $10 \mathrm{~K}$, magnetic cycling experiments we performed on demagnetized samples in fields between $10 \mathrm{mT}$ and $1 \mathrm{~T}$ using a SQUID magnetometer (MPMS3: Quantum Design) in sweeping modes with rate of $5 \mathrm{~K} / \mathrm{min}$ in the range between 50 to $300 \mathrm{~K}$ and $1 \mathrm{~K} / \mathrm{min}$ in the range between 50 and $10 \mathrm{~K}$. The analysis of the dynamic magnetization properties comprises the measurement of the amplitude dependence of the $a c$ susceptibility at $300 \mathrm{~K}$ and ferromagnetic resonance (FMR) spectroscopy. The former measured by a physical property measurement system (PPMS Quantum Design) is used to test the presence of domain walls and the latter to analyze interaction and anisotropy properties ${ }^{48}$. The temperature dependence of the FMR spectra were recorded between 300 and $10 \mathrm{~K}$ and the angular dependence at $300 \mathrm{~K}$ and $10 \mathrm{~K}$ on flakes embedded in paraffin. The resonance field $\left(H_{\text {res }}\right)$, defined as the maximum adsorption, and the linewidth $(\Delta H)$ as peak to peak distance in the first derivative absorption spectra are the key parameters directly obtained from the measurements, and the $g$-value was calculated using the resonance equation $\hbar \nu=g \mu_{B} H_{\text {res }}$ where $\hbar$ is the Planck's constant, $v$ is the microwave frequency, $\mu_{\mathrm{B}}$ is the Bohr's magneton and $g$ the effetive $g$-factor ${ }^{49}$. Moreover, the symmetry ratio $A_{\text {ratio }}=H_{\text {high }} / H_{\text {low }}$ was determined, where the distances between $H_{\text {res }}$ and the corresponding fields of the peak positions define $H_{\text {low }}$ and $H_{\text {high }}$, respectively ${ }^{39}$. The FMR experiments were performed on $\mathrm{X}$-band Bruker ElexSys E500 spectrometer equipped with a goniometer and a cryostat. A sweeping field between 5 and $650 \mathrm{mT}$, microwave frequency between 9.37 and $9.87 \mathrm{GHz}$ depending on the experiment, a power of 0.2 $\mathrm{mW}$, a modulation amplitude of $0.1 \mathrm{mT}$ were used.

Micromagnetic simulation. The flake was modelled as platelets consisting of intergrown crystallites with a size of $20 \mathrm{~nm}$. It has a side length of $200 \mathrm{~nm}$ and a thickness of $20 \mathrm{~nm}$, and the crystallites were generated by means of Voronoi tessellation. The total energy density of the system, in the continuum approximation, is

$$
\varepsilon=A(\nabla \mathbf{m})^{2}+K\left(\alpha^{2} \beta^{2}+\beta^{2} \gamma^{2}+\gamma^{2} \alpha^{2}\right)-\mu_{0} M_{s} \mathbf{H} \cdot \mathbf{m}-\frac{1}{2} \mu_{0} M_{s} \mathbf{H}_{\mathrm{dm}} \cdot \mathbf{m},
$$

where $A$ is the exchange stiffness, $\mathbf{m}$ is the magnetization unit vector $\mathbf{m}=\mathbf{M} / M_{\mathrm{s}}$ with the saturation magnetization $M_{\mathrm{s}}, K$ is the first-order cubic magnetocrystalline anisotropy constant with the directional cosines $\alpha, \beta, \gamma, \mathbf{H}$ is the external field and $\mathbf{H}_{\mathrm{dm}}$ is the local demagnetizing field due to dipole-dipole interactions. The material parameters were taken from the literature $24,26,27,50$ to be $A=2 \times 10^{-12} \mathrm{~J} / \mathrm{m}, K=-1.7 \times 10^{4} \mathrm{~J} / \mathrm{m}^{3}$, and $M_{s}=2.7 \times 10^{5}$ $\mathrm{A} / \mathrm{m}$. These parameters yield an exchange length of $\delta=\sqrt{2 A / \mu_{0} M_{\mathrm{s}}^{2}} \approx 6.6 \mathrm{~nm}$. The system was discretized in finite-difference cells with a dimension of $1 \mathrm{~nm}$. The computation of hysteresis curves was performed for different flake directions from normal to in-plane in $5^{\circ}$ steps using the software package Mumax $3^{51}$ with the steepest conjugate gradient method, and the magnetization was computed in field-cycles between $\mu_{0} H= \pm 1 \mathrm{~T}$. The convoluted and averaged curves were taken to predict bulk properties of a flake.

Received: 8 September 2020; Accepted: 2 December 2020

Published online: 04 February 2021

\section{References}

1. Skinner, B. J., Erd, R. C. \& Grimaldi, F. S. Greigite, the thio-spinel of iron; a new mineral. Am. Miner. 49, 543-555 (1964).

2. Li, W. et al. Formation of greigite $\left(\mathrm{Fe}_{3} \mathrm{~S}_{4}\right)$ in the sediments of saline lake Lop Nur, northwest China, and its implications for paleoenvironmental change during the last 8400 years. J. Asian Earth Sci. 174, 99-108 (2019).

3. Igarashi, K., Yamamura, Y. \& Kuwabara, T. Natural synthesis of bioactive greigite by solid-gas reactions. Geochim. Cosmochim. Acta 191, 47-57 (2016).

4. Vasiliev, I. et al. Early diagenetic greigite as a recorder of the palaeomagnetic signal in Miocene-Pliocene sedimentary rocks of the Carpathian foredeep (Romania). Geophys. J. Int. 171, 613-629 (2007).

5. Hu, S., Appel, E., Hoffmann, V., Schmahl, W. W. \& Wang, S. Gyromagnetic remanence acquired by greigite $\left(\mathrm{Fe}_{3} \mathrm{~S}_{4}\right)$ during static three-axis alternating field demagnetization. Geophys. Int. J. 134, 831-842 (1998).

6. Snowball, I. F. The detection of single-domain greigite $\left(\mathrm{Fe}_{3} \mathrm{~S}_{4}\right)$ using rotational remanent magnetization (RRM) and the effective gyro field (Bg): Mineral magnetic and palaeomagnetic applications. Geophys. Int. J. 130, 704-716 (1997).

7. Cao, F., et al. $3 \mathrm{D} \mathrm{Fe}_{3} \mathrm{~S}_{4}$ flower-like microspheres: High-yield synthesis via a biomolecule-assisted solution approach, their electrical, magnetic and electrochemical hydrogen storage properties. Dalton Trans. 9246-9252 (2009).

8. Li, G. et al. High-purity $\mathrm{Fe}_{3} \mathrm{~S}_{4}$ greigite microcrystals for magnetic and electrochemical performance. Chem. Mater. 26, 5821-5829 (2014).

9. Wu, M., John, S. T. \& Pan, Y. Electronic structures of greigite $\left(\mathrm{Fe}_{3} \mathrm{~S}_{4}\right)$ : A hybrid functional study and prediction for a Verwey transition. Sci. Rep. 6, 21637 (2016).

10. He, Q., Huang, C. \& Liu, J. Preparation, characterization and antibacterial activity of magnetic greigite and $\mathrm{Fe}_{3} \mathrm{~S}_{4} / \mathrm{Ag}$ nanoparticles. Nanosci. Nanotechnol. Lett. 6, 10-17 (2014).

11. Valdez-Grijalva, M. A. et al. Magnetic vortex effects on first-order reversal curve (FORC) diagrams for greigite dispersions. Earth Planet Sci. Lett. 501, 103-111 (2018).

12. Zhang, B., De Wijs, G. \& De Groot, R. Switchable Fermi surface sheets in greigite. Phys. Rev. B 86, 020406 (2012).

13. Roldan, A. et al. Bio-inspired $\mathrm{CO}_{2}$ conversion by iron sulfide catalysts under suitable conditions. Chem. Commun. 51, 7501-7504 (2015).

14. Chang, Y.-S., Savitha, S., Sadhasivam, S., Hsu, C.-K. \& Lin, F.-H. Fabrication, characterization, and application of greigite nanoparticles for cancer hyperthermia. J. Colloid Interf. Sci. 363, 314-319 (2011). 
15. Just, J. et al. Environmental control on the occurrence of high-coercivity magnetic minerals and formation of iron sulfides in a 640 ka sediment sequence from Lake Ohrid (Balkans). Biogeosciences 13, 2093-2103 (2016).

16. Rowan, C. J., Roberts, A. P. \& Broadbent, T. Reductive diagenesis, magnetite dissolution, greigite growth and paleomagnetic smoothing in marine sediments: A new view. Earth Planet. Sci. Lett. 277, 223-235 (2009).

17. Florindo, F. \& Sagnotti, L. Palaeomagnetism and rock magnetism in the upper Pliocene Valle Ricca (Rome, Italy) section. Geophys. J. Int. 123, 340-354 (1995).

18. Bazylizinki, D. A., Heywood, B. R., Mann, S. \& Frankel, R. B. $\mathrm{Fe}_{3} \mathrm{O}_{4}$ and $\mathrm{Fe}_{3} \mathrm{~S}_{4}$ in a bacterium. Nature 366, 218 (1993).

19. Lefèvre, C. T. et al. A cultured greigite-producing magnetotactic bacterium in a novel group of sulfate-reducing bacteria. Science 334, 1720-1723 (2011).

20. Chen, A. et al. Magnetic properties of uncultivated magnetotactic bacteria and their contribution to a stratified estuary iron cycle. Nat. Commun. 5, 1-11 (2014).

21. Rhodes, J. M., Jones, C. A., Thal, L. B. \& Macdonald, J. E. Phase-controlled colloidal syntheses of iron sulfide nanocrystals via sulfur precursor reactivity and direct pyrite precipitation. Chem. Mater. 29, 8521-8530 (2017).

22. Feng, M. et al. Bioinspired greigite magnetic nanocrystals: Chemical synthesis and biomedicine applications. Sci. Rep. 3, 2994 (2013).

23. He, Z., Yu, S. H., Zhou, X., Li, X. \& Qu, J. Magnetic-field-induced phase-selective synthesis of ferrosulfide microrods by a hydrothermal process: Microstructure control and magnetic properties. Adv. Funct. Mater. 16, 1105-1111 (2006).

24. Winklhofer, M., Chang, L. \& Eder, S. H. On the magnetocrystalline anisotropy of greigite $\left(\mathrm{Fe}_{3} \mathrm{~S}_{4}\right)$. Geochem. Geophys. Geosyst. 15, 1558-1579 (2014).

25. Devey, A., Grau-Crespo, R. \& de Leeuw, N. Electronic and magnetic structure of $\mathrm{Fe}_{3} \mathrm{~S}_{4}$ : GGA+ U investigation. Phys. Rev. B 79, 195126 (2009).

26. Chang, L. et al. Magnetic structure of greigite $\left(\mathrm{Fe}_{3} \mathrm{~S}_{4}\right)$ probed by neutron powder diffraction and polarized neutron diffraction. J. Geophys. Res. 114, B07101 (2009).

27. Chang, L. et al. Fundamental magnetic parameters from pure synthetic greigite $\left(\mathrm{Fe}_{3} \mathrm{~S}_{4}\right)$. J. Geophys. Res. 113, B06104 (2008).

28. Lyubutin, I. et al. Magnetic, structural, and electronic properties of iron sulfide $\mathrm{Fe}_{3} \mathrm{~S}_{4}$ nanoparticles synthesized by the polyol mediated process. J. Nanopart. Res. 15, 1397 (2013)

29. Muscas, G. et al. The interplay between single particle anisotropy and interparticle interactions in ensembles of magnetic nanoparticles. Phys. Chem. Chem. Phys. 20, 28634-28643 (2018).

30. Koulialias, D. et al. Competitive and cooperative anisotropy in magnetic nanocrystal chains of magnetotactic bacteria. J. Appl. Phys. 120, 083901 (2016).

31. Paolella, A. et al. Charge transport and electrochemical properties of colloidal greigite $\left(\mathrm{Fe}_{3} \mathrm{~S}_{4}\right)$ nanoplatelets. Chem. Mater. 23, 3762-3768 (2011).

32. Roberts, A. P., Chang, L., Rowan, C. J., Horng, C. S. \& Florindo, F. Magnetic properties of sedimentary greigite $\left(\mathrm{Fe}_{3} \mathrm{~S}_{4}\right): \mathrm{An}_{\mathrm{n}} \mathrm{update}$ Rev. Geophys. 49, RG1002 (2011).

33. Goya, G. F., Berquo, T., Fonseca, F. \& Morales, M. Static and dynamic magnetic properties of spherical magnetite nanoparticles. J. Appl. Phys. 94, 3520-3528 (2003).

34. Coey, J., Spender, M. \& Morrish, A. The magnetic structure of the spinel $\mathrm{Fe}_{3} \mathrm{~S}_{4}$. Solid State Commun. 8, 1605-1608 (1970).

35. Koulialias, D. et al. The Besnus transition in single-domain 4C Pyrrhotite. Geochem. Geophys. Geosyst. 20, 5216-5224 (2019).

36. Cojocaru, S., Naddeo, A. \& Citro, R. Modification of the Bloch law in ferromagnetic nanostructures. Europhys. Lett. 106, 17001 (2014).

37. Della Torre, E., Bennet, L. H. \& Watson, R. E. Extension of the Bloch $T^{3 / 2}$ law to magnetic nanostructure: Bose-Einstein condensation. Phys. Rev. Lett. 94, 147210 (2005).

38. Chang, L. et al. Low-temperature magnetic properties of greigite (Fe3S4). Geochem. Geophys. Geosys. 10, Q01Y04 (2009).

39. Weiss, B. P. et al. Ferromagnetic resonance and low-temperature magnetic tests for biogenic magnetite. Earth Planet. Sci. Lett. 224, 73-89 (2004).

40. Chang, L. et al. Ferromagnetic resonance characterization of greigite $\left(\mathrm{Fe}_{3} \mathrm{~S}_{4}\right)$, monoclinic pyrrhotite $\left(\mathrm{Fe}_{7} \mathrm{~S}_{8}\right)$, and non-interacting titanomagnetite $\left(\mathrm{Fe}_{3-\mathrm{x}} \mathrm{Ti}_{\mathrm{x}} \mathrm{O}_{4}\right)$. Geochem. Geophys. Geosyst. 13, 0541 (2012).

41. Gehring, A. U., Fischer, H., Louvel, M., Kunze, K. \& Weidler, P. G. High temperature stability of natural maghemite: A magnetic and spectroscopic study. Geophys. J. Int. 179, 1361-1371 (2009).

42. Polder, D. \& Smit, J. Resonance phenomena in ferrites. Rev. Mod. Phys. 25, 89-90 (1953).

43. Gehring, A. U., Fischer, H., Charilaou, M. \& García-Rubio, I. Magnetic anisotropy and Verwey transition of magnetosome chains in Magnetospirillum gryphiswaldense. Geophys. J. Int. 187, 1215-1221 (2011).

44. Bruker AXS TOPAS V6: General profile and structure analysis software for powder diffraction data. - User's Manual; Bruker AXS, Karlsruhe, Germany (2017).

45. Young R.A. The Rietveld Method. International Union of Crystallography (1995)

46. Roberts, A. P., Pike, C. R. \& Verosub, K. L. First-order reversal curve diagrams: A new tool for characterizing the properties of natural samples. J. Geophys. Res. 105, 28461-28475 (2000).

47. Harrison, R. J. \& Feinberg, J. M. FORCinel: An improved algorithm for calculating first-order reversal curve distributions using locally weighted regression smoothing. Geochem. Geophys. Geosyst. 9, Q05016 (2008).

48. Charilaou, M. et al. Evolution of magnetic anisotropy and thermal stability during nanocrystal-chain growth. Appl. Phys. Lett. 99, 182504 (2011).

49. Vonsovskii, S.V. (ed.). Ferromagnetic Resonance: The Phenomenon of Resonant Absorption of a High-Frequency Magnetic Field in Ferromagnetic Substances. (Elsevier, Amsterdam, 1966).

50. Valdez-Grijalva, M. A. et al. Micromagnetic simulatins of first order reversial curve (FORC) diagrams of framboidal greigite. Geophys. Int. J. 222, 1126-1134 (2020).

51. Vansteenkiste, A. et al. The design and verification of MuMax3. AIP Adv. 4, 107133 (2014)

\section{Acknowledgements}

The authors thank Severian Gvasaliya helping with the MPMS measurements and René Tschaggelar with the FMR experiments at ETH Zürich. The project was supported by Swiss National Sciences Foundation Grant no. 200021_165851 and JMR and JEM by the US National Science Foundation CHE-1905265, and the US Department of Education graduate assistance in areas of national need (GAANN) fellowship P200A140248.

\section{Author contributions}

A.U.G. conceived the study and organized the research. J.M.R., J.E.M and P.G.W. synthesized and characterized the greigite nanoparticles, B.L. and D.K. provided the magnetic and B.L. the FMR measurements, A.U.G., B.L., D.K., and M.C. analysed the magnetic data, M.C. performed the micromagnetic simulations. A.U.G. and M.C. wrote the final version of the paper and all authors discussed and commented on it. 


\section{Competing interests}

The authors declare no competing interests.

\section{Additional information}

Correspondence and requests for materials should be addressed to A.U.G.

Reprints and permissions information is available at www.nature.com/reprints.

Publisher's note Springer Nature remains neutral with regard to jurisdictional claims in published maps and institutional affiliations.

(c) (1) Open Access This article is licensed under a Creative Commons Attribution 4.0 International License, which permits use, sharing, adaptation, distribution and reproduction in any medium or format, as long as you give appropriate credit to the original author(s) and the source, provide a link to the Creative Commons licence, and indicate if changes were made. The images or other third party material in this article are included in the article's Creative Commons licence, unless indicated otherwise in a credit line to the material. If material is not included in the article's Creative Commons licence and your intended use is not permitted by statutory regulation or exceeds the permitted use, you will need to obtain permission directly from the copyright holder. To view a copy of this licence, visit http://creativecommons.org/licenses/by/4.0/.

(C) The Author(s) 2021 